\title{
BAILANDO EL SILENCIO: LA SIGNODANZA
}

\author{
Rakel Rodríguez Ruiz \\ Universidad de Granada. Licenciada en Pedagogía \\ Compañía Arymux
}

\section{Resumen}

La SignoDanza es la disciplina con la que se trabaja en la compañía Arymux y que consiste en la fusión de distintos estilos de danza con las lenguas de signos. Bailando el silencio es el nombre de un espectáculo de SignoDanza de la compañía.

La investigación que estamos realizando de las múltiples posibilidades creativas, expresivas y de comunicación que ofrece la SignoDanza, parte de una perspectiva que permite a los/ as bailarines/as con o sin discapacidad, integrar la música y las palabras a través de la lengua de signos en su movimiento; es una forma de fusionar estilos, públicos y lenguajes diferentes.

Un modo de bailar el silencio, que, a su vez, está lleno de contenido, de emociones y de palabras.

Palabras clave: LENGUA DE SIGNOS; SIGNODANZA; ARYMUX, FUSIÓN; INTEGRACIÓN

\section{Abstract}

SignDance is a discipline Arymux company works with and it is a fusion between different dance styles and sign languages. "Dancing the Silence" is the name for this company signdance performance.

The research is being done in the different creative, expresive and comunicative posibilities that Signdance offers, it is a part of the perspective that allows the dancers with or without disability, to integrate music and words through sign language in movement. This new concept of dance enables us to put together different styles, languages and audiences.

Signdance is a way to dance the silence, a silence full of contents, emotions and words.

Keywords: SIGN LANGUAGE; SIGNDANCE; ARYMUX; FUSION; INTEGRATION

\footnotetext{
Rodríguez Ruiz, Rakel. 2015. "Bailando el silencio: La SignoDanza". AusArt 3 (1): 118-129. D0I: 10.1387/ausart.14424
}

\section{AUSART}




\section{LENGUA DE SIGNOS Y MOVIMIENTO}

La lengua de signos es la lengua natural de las personas sordas. Es la lengua que les permite comunicarse en cualquier ámbito y expresar cualquier sentimiento o idea. Son lenguas con carácter viso-gestual, que utilizan el espacio y el movimiento para materializarse. Desde el punto de vista artístico, siempre he considerado que es una forma de crear palabras con las manos, de dibujarlas en el aire a través de pautas concretas y movimientos secuenciados. Visto desde fuera, en muchas ocasiones, recuerda a una coreografía realizada con la parte superior del cuerpo. Ese fue el motor que me llevó a desarrollar un trabajo de investigación cuyo origen era el movimiento que nace de las diferentes lenguas de signos y que pretendía fusionar con los distintos estilos de danza, realzando el significado y la expresión de los mismos.

Según Baker y Cokely (1980) "las lenguas son sistemas de signos perfectamente organizados que se transforman en el tiempo, y que los miembros de una comunidad acuerdan y usan para interactuar entre ellos, expresar sus ideas, emociones e intenciones y para transmitir su cultura de una generación a otra".

La lengua de signos se utilizó desde un primer momento dentro de la compañía como elemento comunicativo y a su vez, como herramienta creadora desde el movimiento. Para desarrollar las ideas que queremos exponer en este artículo, es importante mencionar que la lengua de signos no es universal. Cada país tiene su propia lengua de signos. Incluso existe un sistema de signos internacional, equivalente al esperanto en las lenguas orales, que es útil para la comunicación entre personas sordas de distintos países, pero no es considerado una lengua. En el libro Interpretación del Sistema de Signos Internacional $(2000,44)$ se dice: "el principal argumento para desechar el hecho de que el sistema de signos internacional sea una lengua es que nadie lo tiene como lengua materna, no utilizándose en la convivencia diaria y no es utilizado para transmitir la cultura de una generación a otra"

Pero desde que aprendí a dominar este sistema de signos, fui consciente de que aporta elementos sumamente expresivos, y a través de ellos, nos valemos en la compañía del mismo para desarrollar el proceso coreográfico y de fusión entre la danza y la lengua de signos, sobre todo cuando el montaje coreográfico tiene carácter internacional, para conseguir hacerlo aún más accesible al público de cualquier país. En el mismo libro mencionado en el párrafo anterior, 


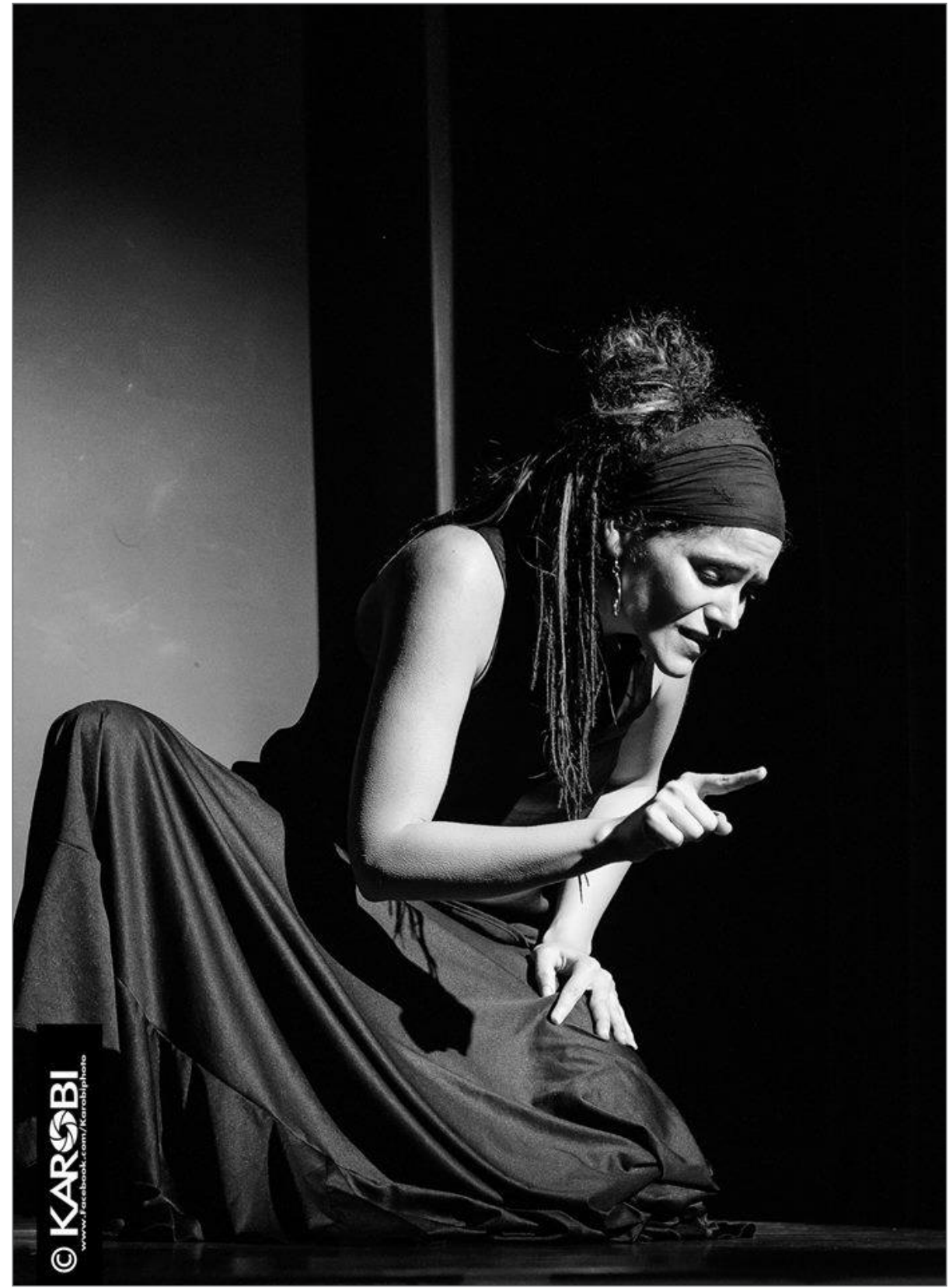

Figura1.- SignoDanza. Fotografía: Karob 
$(2000,28)$ encontramos: "podríamos decir que los signos internacionales que se desarrollaron de una forma natural a través del contacto entre personas usuarias de distintas lenguas de signos han perdurado de alguna manera los dos últimos siglos"

Mi trabajo a lo largo de los casi 20 años que llevo trabajando directamente con el colectivo de personas sordas, en cualquiera de sus ámbitos y la experiencia adquirida a través de la interpretación de la lengua de signos aplicada al ámbito artístico, me condujo a la necesidad de plantear una metodología que sentara las bases de unas pautas concretas que posibilitaran la fusión real de la lengua de signos con la práctica de la danza. Por un lado, como cauce de comunicación y expresión personal, y por otro, como elemento creador que integrara la música, la danza y la lengua de signos.

Teniendo en cuenta que la base del trabajo que realizamos con el movimiento parte de un idioma, una lengua, es fundamental su dominio completo, conocer a fondo su lingüística y sus diferentes maneras de habitar el espacio. A partir de ahí, se puede llegar a la creación coreográfica con bailarines que no sean personas sordas ni signantes de este idioma. Para ellos es importante que las pautas de movimiento estén estructuradas y que el trabajo sea supervisado metódicamente para traducirlo de una forma clara en secuencias o partituras que conjuguen los movimientos de los signos con las amplias posibilidades de expresión del cuerpo. Eugenio Barba $(1994,70)$ dice "muchas incomprensiones nacen del lenguaje que se elige para transmitir algunas experiencias técnicas que son claras y concretas en la acción"

\section{DEL SIGNO A LA DANZA. Y VICEVERSA}

La formación pedagógica, la experiencia como intérprete de lengua de signos adquirida y el trabajo de docencia en danza con personas sordas y oyentes, fueron pilares básicos para mí cuando me planteé trabajar en una disciplina que me permitiera viajar desde los signos al movimiento y viceversa. La SignoDanza es la esencia de la compañía, la base para crear espectáculos como "Bailando el silencio", integradores de lenguajes, públicos y modos de expresión. 
Según Alicia Gómez $(2014,242)$, "la práctica de la danza no sólo nos permite aprender danza sino también reflexionar, crear, expresar y comunicar sentimientos, pensamientos y relatos de una forma consciente, creativa, cooperativa y estética. Además, puede contribuir al autoconocimiento, a la automotivación, a la resolución de problemas, al bienestar psicológico y al desarrollo motriz, cognitivo y socioemocional de las personas".

En este sentido, uno de los objetivos principales en los proyectos de la compañía es hacerlos accesibles a todos los públicos, eliminando al máximo posible las barreras de comunicación con las que las personas sordas, sordociegas o con otro tipo de discapacidad, se encuentran al intentar acceder a la oferta cultural existente. Teniendo en cuenta que las personas integrantes de la compañía en los diferentes cursos, proyectos y espectáculos que se realizan pueden o no ser personas con discapacidad, el autoconocimiento, la cooperación, la reflexión sobre el trabajo que realizamos a diario, el desarrollo personal, grupal y artístico y la comunicación de las emociones y sentimientos con los que queremos trabajar, constituyen los cimientos de Arymux y de todos los procesos creativos que llevamos a cabo.

Según Oliver Sacks $(1997,103)$ "El lenguaje y el pensamiento siempre son personales: lo que decimos nos expresa, igual que nuestro lenguaje interior".

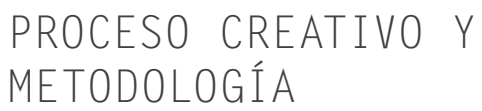

No existen referencias bibliográficas ni materiales previos en el trabajo con SignoDanza, más que el que estamos desarrollando dentro de la compañía. Eso dificulta el trabajo de creación y de intercambio, sobre todo cuando se siente la necesidad de referentes externos que nos acompañen en los proyectos. Aún así, desde un primer momento consideré que estar abiertos a la experiencia que nos pueden proporcionar las diferentes artes escénicas y los profesionales que trabajan en ellas en cualquiera de sus formas es la clave para avanzar. Por ese motivo, desde el comienzo, la compañía trabaja con personas de distinta nacionalidad y procedencia, personas con y sin discapacidad, actores y actrices, intérpretes de lengua de signos, profesionales del mimo y de la expresión corporal y bailarines de distintas disciplinas. Todos estos profesionales aportan perspectivas diferentes y complementarias, que 
son necesarias para crear proyectos y espectáculos donde la diversidad y la calidad artística puedan crecer por igual. Con ese objetivo de integrar toda esa variedad de estilos y conocimientos, y de integrarlos en un montaje, surgió el espectáculo "Bailando el silencio".

El proceso de trabajo que desarrollamos en él y en el resto de espectáculos de la compañía, es complejo. Para trabajar la expresión corporal y facial dentro de una coreografía, se buscan los medios a través de los cuales se puedan percibir las vibraciones del sonido, con elementos que permitan a las personas sordas integrantes del montaje, seguir el ritmo y apreciar las diferentes intensidades del sonido que perciben las personas oyentes. Cuando se coreografían textos a partir de la lengua de signos, o piezas musicales con letra, realizamos previamente al trabajo de movimiento, una traducción e interpretación del contenido que se pretende transmitir. Para hacer una buena interpretación de esos mensajes y de todos los elementos musicales y de contenido que los componen, es necesario llevar a cabo un proceso metodológico muy estricto. Por un lado hay que analizar el mensaje y realizar la traducción desde la lengua oral en la que esté dicha composición, a la lengua de signos con la que estemos trabajando. Por otro hay que analizar el ritmo y la melodía para poder adaptarlos y sincronizarlos con el texto, de manera que se llegue a transmitir el movimiento y la intensidad necesaria, así como adoptar el registro adecuado para transmitir el mensaje.

Rakel Rodríguez (2014) "Utilizando la lengua de signos como vehículo de comunicación y como medio para crear, se puede conseguir una coreografía que, contemplada por una persona que no domine la lengua de signos, se muestra como una partitura de movimiento en la que se intuyen palabras danzando en el espacio a través del cuerpo. Contemplada por una persona sorda u oyente que domine la lengua de signos, puede además llegarse a entender el contenido del mensaje que se está transmitiendo a través de esos movimientos.

Pilar Lara y Esther de los Santos $(1999,48)$ son profesionales de la interpretación de lengua de signos que tengo como referencia en muchos de los trabajos que se realizan desde la compañía, teniendo en cuenta que el primer paso para el trabajo con la lengua de signos y el mensaje que queremos transmitir, es realizar una buena interpretación del mismo. Ellas enuncian una serie de 
requisitos que permiten que ese proceso de interpretación se lleve a cabo con calidad. Son los siguientes:

1. El intérprete percibe y entiende el mensaje en una lengua, extrayendo su significado de la entonación, las palabras, comportamiento no verbal, pausas o cualquier otro mecanismo que pueda facilitar alguna clave para su análisis.

2. Elimina, de forma inmediata y deliberada, todo el revestimiento lingüístico del mensaje para quedarse con una representación mental del mismo (un concepto o idea).

3. Reproduce el contenido del mensaje en la otra lengua, intentando, por un lado, expresar de forma completa el mensaje original y, por otro, hacerlo de una forma comprensible para el destinatario del mismo. Además, el intérprete deberá ir comprobando las reacciones del receptor, lo que le servirá para confirmar que el mensaje está llegando de forma inteligible.

Teniendo en cuenta esto, en la SignoDanza nos encontramos con un proceso creativo más amplio aún que el propio proceso de interpretación. Esto se debe a que necesitamos crear una coreografía que transmita todos esos elementos y que además esté fusionada con el movimiento de todo el cuerpo.

Aún así, en la mayoría de las ocasiones el proceso creativo no está tan supeditado a la palabra como pueda parecer. La abstracción del mensaje, la simplicidad de los movimientos y la capacidad creativa de los bailarines son pautas que siempre tenemos presentes para no encorsetar y limitar el trabajo y las propuestas que puedan realizarse.

Etienne Decroux, $(1963,67)$ dice: “¿danzamos cuando elevamos los ojos? Y cuado éstos no pueden levantarse más, ¿danzamos elevando la cabeza? NO. Es la mirada que sigue su curso. Pero si se quiere seguir viendo al que se aleja, hace falta girar el cuello hacia atrás y luego sucesivamente pecho, cintura y pelvis... ¿En dónde comienza la danza? ¿En el cuello o en el muslo?"

Trabajar con diferentes lenguas de signos, aprovechando la variedad lingüística que nos ofrecen, e incorporar variaciones en el movimiento de cada uno de los signos, nos llevó en el montaje "Bailando el silencio" a elegir un estilo de danza para cada bailarín, y a partir de ahí, respetando su manera de enten- 


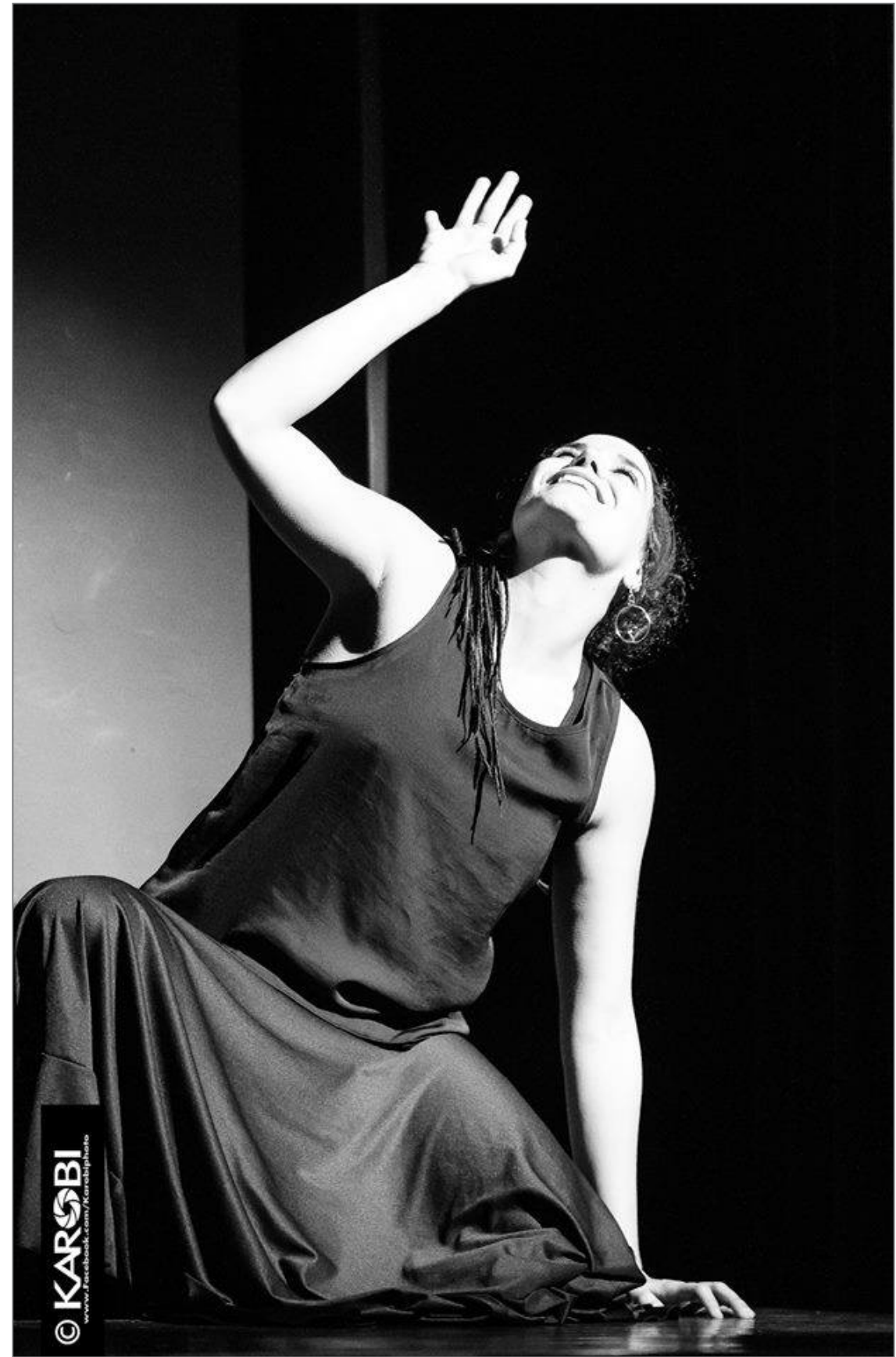

Figura 2. SignoDanza. Fotografía: Karobi. 
der el cuerpo y el movimiento, empezar a fusionar la dirección y la intensidad de cada signo con las capacidades expresivas del cuerpo, de la cara, de la mirada, y de todos los registros que se pueden transmitir utilizando la manera individual de bailar. Este proceso de trabajo, me sigue fascinando y demostrando que, a pesar del camino andado, aún estamos al inicio de la investigación y del desarrollo de una técnica que se va construyendo a la vez que se crea.

\section{BAILANDO EL SILENCIO}

Bailando el silencio es, como hemos dicho con anterioridad, un espectáculo de SignoDanza de la compañía Arymux, accesible para todos los públicos, que se ha realizado en diferentes ciudades españolas y también en otros países como Italia y que, como se ha comentado a lo largo del artículo, surge de la investigación y del trabajo realizado a nivel personal con personas con y sin discapacidad y de la colaboración con otros profesionales de la danza y de otras artes escénicas. Es un proyecto que pretende hacer invisibles las barreras que separan a las personas sordas y sordociegas de la música, la danza, el teatro y la cultura en general, hilando redes que contribuyan a disminuir las diferencias.

Pilar Lara y Esther de los Santos (1999, 19): "la interpretación de la lengua de signos surge como respuesta a una demanda social de las personas sordas: la necesidad de romper las barreras de comunicación que las aíslan de la sociedad y el derecho a participar en ésta en igualdad de condiciones con respecto al resto de los ciudadanos"

Es un espectáculo que cambia de contenidos y formas porque va evolucionando con el tiempo y porque fue creado con el fin de acompañar la investigación de esa fusión de las lenguas de signos con diversos estilos de danza. A partir de él surgió la disciplina artística que terminé denominando SignoDanza. "Bailando el silencio" es un engranaje de estilos y movimientos diferentes, por lo que, en cada actuación, para crear cada una de las piezas que lo componen, se tiene en cuenta la necesidad de trabajar con los diferentes parámetros formativos de las lenguas de signos y con lo que aporta cada uno de los estilos de danza, o yendo más allá, con lo que aportan cada uno de los bailarines y demás artistas que ejecutan las piezas, puesto que esa capacidad expresiva 
hay que valorarla de un modo individual. Se tiene en cuenta su manera propia de expresarse y de transmitir las emociones, de transformar los signos y conducirlos de un modo sutil, hacia el movimiento coreográfico.

Otro elemento con el que se trabaja habitualmente en el espectáculo, aparte del cuerpo en sí, y al que se le presta especial atención, es la expresión facial. Porque los registros que utilizamos y transmitimos a través de ella, son la expresión de la voz, de la intensidad y de las emociones de la lengua oral. Nos solemos centrar en el trabajo de la mirada y sus distintas energías a la hora de transmitir sentimientos unidos al signo y al movimiento que se están realizando en cada momento.

El proceso de trabajo en este espectáculo va, por tanto del movimiento al signo, o del signo al movimiento. De la expresión corporal a la palabra. De la palabra a la expresión corporal. De lo abstracto a lo concreto, de lo concreto a lo abstracto. Del significante al significado. Paulina Ossona dice $(1984,22)$ "todo movimiento, desde el mecánico hasta el simbólico, contiene siempre una gran carga expresiva"

Como se ha mencionado en varias ocasiones, en el proceso creativo de este espectáculo me parece fundamental nutrirnos de distintas disciplinas y de lo que cada una puede ofrecer. A su vez, para completar con éxito el trabajo coreográfico se requiere un nivel muy alto de lengua de signos. Un dominio de esta lengua que permita trabajar abstrayendo el mensaje, porque, a veces, es necesario utilizar signos de diferentes lenguas o utilizar el sistema de signos internacional para hacer más claro el movimiento, o simplemente, porque un signo en una lengua es más expresivo que en otra diferente, para lo que se pretende transmitir. Así mismo, hay una exigencia técnica muy alta en el caso de los bailarines y del control del cuerpo cuando se trata de los actores y actrices y de los intérpretes de lengua de signos.

Jacques Lecoq $(2007,233)$ dice "Igual que el mimo se ha abierto al teatro, también se ha abierto ampliamente al movimiento y sobre todo a la danza. Algunos coreógrafos han vuelto a buscar en el teatro gestos que la danza había perdido, renovando así en parte la danza contemporánea gracias a este reencuentro." 


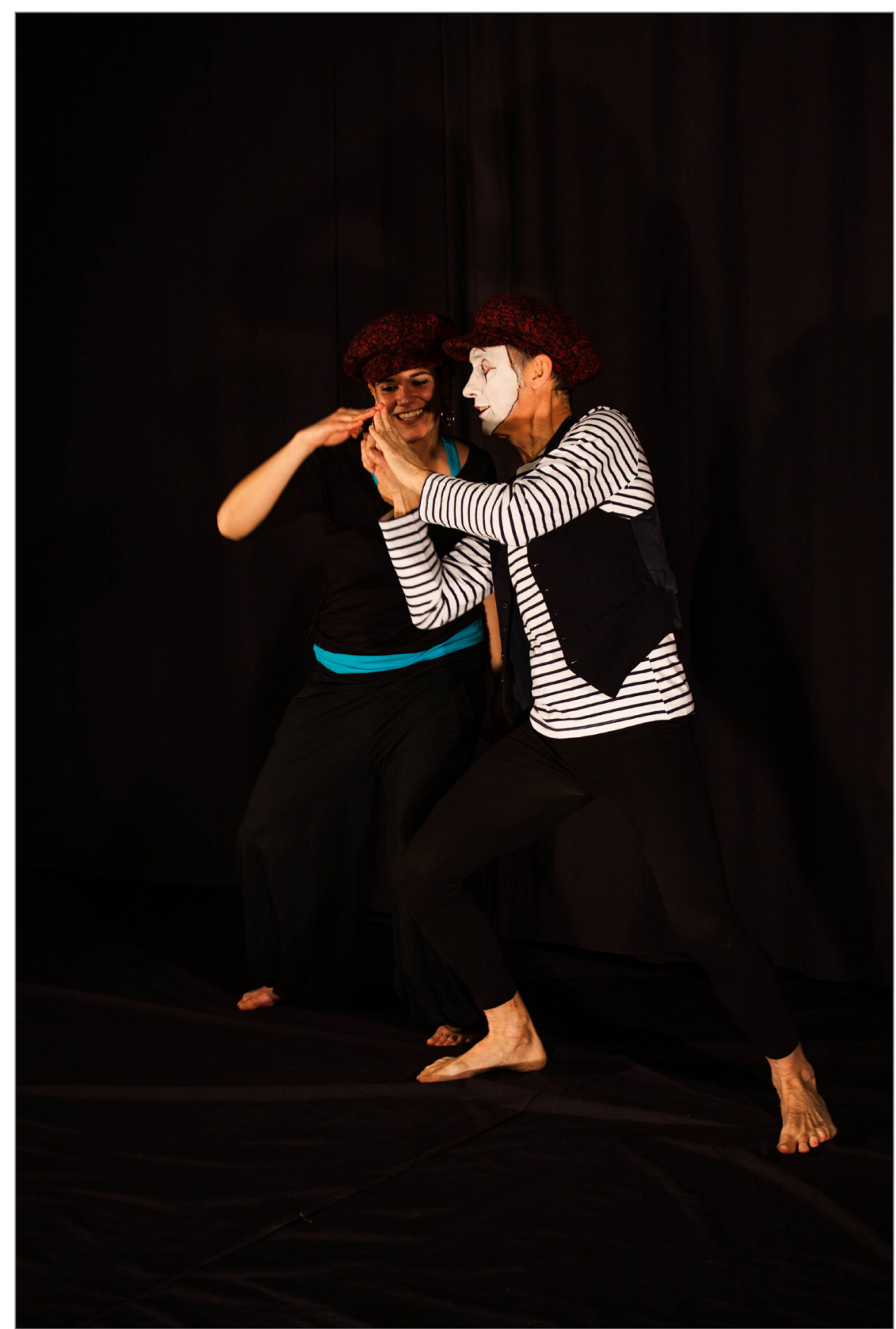

Figura 3. Ensayo Coreografía de SignoDanza y Mimo. Fotografía: Casimiro Ondo. 


\section{CONCLUSIÓN}

El proceso de trabajo con la SignoDanza es continuo y progresivo, como lo es el de cualquier otro ámbito y disciplina artística. Es necesario continuar investigando en equipo junto a profesionales de la danza y otras artes escénicas, las posibilidades infinitas que ofrece la lengua de signos y su fusión con los diferentes estilos. Es un camino en el que estamos inmersos y en el que, muchas veces en solitario y otras acompañados, continuaremos trabajando, experimentando y desarrollando este proceso creativo que, como las lenguas de signos y la danza, está en continua evolución. Terminamos con las palabras de Eugenio Barba $(1994,257)$ "sólo puedo decirles: utilicen su desorientación para descubrir su cara oculta bajo la máscara de la danza. No olviden que su trabajo, su presencia, deben "enamorar". Hemos llegado al final y cada uno de nosotros inicia el retorno a su soledad".

\section{Referencias}

Barba, Eugenio. 1994. La canoa de papel: Tratado de antropología teatral. Argentina: Catálogos

Cokely,Dennis \& Charlotte Baker-Shenk. 1980. American sign language: A teacher's resource text on grammar and culture. Silver Spring, MD: T.J. Publishers

Decroux, Etienne. 1963. Paroles sur le mime. París: Gallimard

Gómez Linares, Alicia. 2014 "Explorando nuevos horizontes para la danza como recurso educativo socioemocional”. Pp. 235-43 en La investigación en Danza en España 2014, III Congreso Nacional "La Investigación en Danza" (Bilbao). Valencia: Mahali

Lara Burgos, Ma del Pilar y Esther de los Santos Rodríguez. 1999. Técnicas de interpretación de Lengua de signos. Madrid: CNSE

Lecoq, Jacques. 2007. El cuerpo poético. Barcelona: Alba

Moreno Rodríguez, Ana, Pilar Pinedo García y Andrés Rodríguez Falquina. 2000. Interpretación del sistema de signos internacional. Madrid: CNSE

Ossona, Paulina. 1984. La educación por la danza: Enfoque metodológico. Barcelona: Paidós

Rodriguez Ruiz, Rakel "La SignoDanza: Palabras en movimiento". Pp. 149-59 en La investigación en Danza en España 2014, III Congreso Nacional "La Investigación en Danza" (Bilbao). Valencia: Mahali

Sacks, Oliver. 1997. Veo una voz: Viaje al mundo de los sordos. Salamanca: Anaya 